\title{
Peter Grünberg (1939-2018)
}

\section{Physicist who revolutionized data storage with work on magnetism in nanomaterials.}

$\mathrm{P}$ eter Grünberg was one of the first physicists to understand the potential of nascent nanotechnologies for fundamental research. He discovered giant magnetoresistance, or GMR: a large change in electrical resistance induced by a small magnetic field in stacks of ultrathin magnetic and non-magnetic layers. For this, he won a share of the 2007 Nobel Prize in Physics (as did I; we independently discovered the same effect). Ultimately, his work led to the development of hard-disk drives and greatly increased data storage. It also kicked off the field of spintronics. Grünberg died on 7 April 2018, aged 78.

Grünberg was born in 1939 in Pilsen, Bohemia, then a German protectorate, now part of the Czech Republic. In 1945, his family left for West Germany. There, at 19, Grünberg went to study physics at the Goethe University Frankfurt; he then did $\mathrm{ahD}$ at the Technical University of Darmstadt.

For his $\mathrm{PhD}$, he used optical spectroscopy to determine the energy levels of rare-earth ions in magnetic garnet crystals. In his postdoc, he turned another spectroscopy technique - Raman scattering - on garnets, this time at Carleton University in Ottawa, Canada, from 1969 to 1971 . And in 1972, thanks to his expertise in the spectroscopic study of magnetic materials, Grünberg was offered a post at the newly founded Institute for Magnetism at the Jülich Research Centre in Germany.

Here, Grünberg quickly demonstrated his pioneering spirit, developing the spectroscopy technique of Brillouin light-scattering spectroscopy (BLS). BLS examines the inelastic scattering of light; it can probe both the ground state of magnetic materials and their excited states. In the 1970s, physicists were struggling to pick up the specific excitation modes expected to occur at the surface of magnetic materials. Grünberg singled out these modes, and identified them as spin waves of the Damon-Eshbach type.

During a sabbatical at Argonne National Laboratory in Illinois in 1985, Grünberg used an emerging technique of growing metals on single crystals to extend his BLS experiments to layers of magnetic materials less than 1 nanometre thick. This led to his first major discovery. In a sandwich of magnetic iron, non-magnetic chromium and more iron, he and his co-workers demonstrated the existence of antiferromagnetic exchange coupling between the iron layers across chromium (P. Grünberg et al. Phys. Rev. Lett. 57, 2442; 1986). This was the

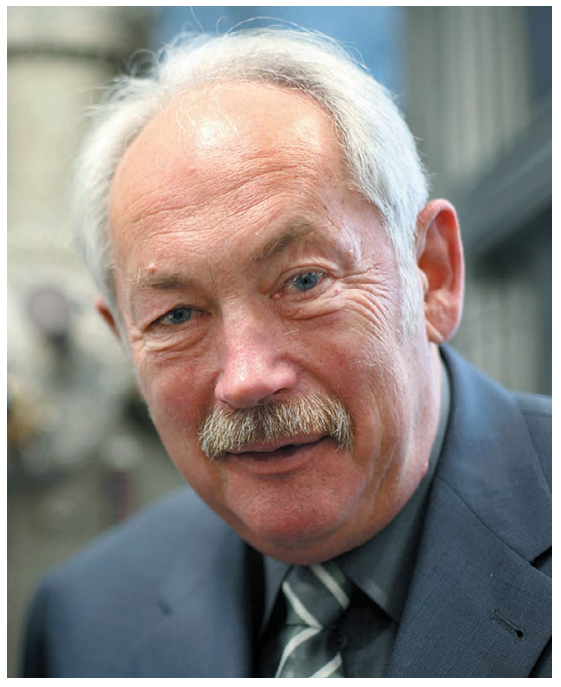

first demonstration of a quantum effect in magnetism. The coupling results from the interference between electronic wave functions reflected at the surface of the magnetic layers. For me, it was also the revelation of a nanostructure in which I could test some of my own ideas about magnetoresistance.

Soon after, in early 1988, our two teams independently discovered GMR. Grünberg's group showed it in their iron-chromiumiron sandwich; my team in France, in a stack of 40 layers of iron and chromium.

It was clear from the outset that GMR would have vast applications - especially because it could happen at room temperature. It could be used to detect small magnetic fields easily, which proved useful for magnetic sensors and, in particular, for reading magnetic hard disks. It was also the first example of electronics that exploit both the spin and charge of electrons - a field today called spintronics.

I met Grünberg in August 1988 at the International Colloquium on Magnetic Films and Surfaces in Le Creusot, a small town in the middle of France. We presented our respective results, and came to the conclusion that we had made the same discovery. To our delight, most participants seemed to think it was important: we celebrated with a couple of glasses of local wine, a Pommard burgundy. That night, we had the feeling that the conference concert, with pieces played on piano and violin by some of our colleagues, was in celebration of our work. Grünberg, a skilled guitarist, did not play that time, but I had several opportunities to hear him play.

Grünberg had an impressive vision for smart GMR-based devices, including the spin-valve concept that he patented and was later developed at IBM for use in hard disks. The first commercial GMR-based hard disks appeared in 1997. Since then, their datastorage capacity has risen by almost three orders of magnitude. Grünberg conceived of many other devices, from magnetic sensors to the compass used in smartphones today.

The discovery of GMR kicked off an intense period of research activity, including experiments on GMR and interlayer exchange coupling in a great diversity of magnetic multilayers. At the same time, the theory behind GMR was developed. Grünberg's Jülich team took a semi-classical approach; I worked in collaboration with New York University on a quantum approach.

The burgeoning field of spintronics has yielded fascinating results. In 1995, Terunobu Miyazaki and Nobuki Tezuka in Japan, and a US group led by Jagadeesh Moodera, independently showed that quantum tunnelling of electrons between magnetic layers gives rise to much larger magnetoresistances than with GMR (T. Miyazaki and N. Tezuka J. Magn. Magn. Mat. 139, L231-L234; 1995; J. S. Moodera et al. Phys. Rev. Lett. 74, 3273; 1995). In 1996, John Slonczewski at IBM in Yorktown Heights, New York, and Luc Berger at Carnegie Mellon University in Pittsburgh, Pennsylvania, introduced the concept that transferring spins between magnetic materials could create torque on their magnetization direction (J. C. Slonczewski J. Magn. Magn. Mat. 159, L1-L7; 1996; L. Berger Phys. Rev. $B$ 54, 9353; 1996). Today, this mechanism is exploited to write non-volatile magnetic memories. Grünberg and his team contributed much to these research fields.

Grünberg was warmly esteemed by colleagues around the world for his great creative talent in physics, and for his integrity and modesty. To me, he was also a good friend. And I liked his sense of humour. Having shared emotional and amusing moments in Stockholm, we often recalled our procession to the Nobel banquet, when neither of us was completely successful in our attempts not to tread on the trains of the Royal Princesses.

Peter was a great physicist, and a gentle and sincere person. The spintronics and nanomagnetism community will miss him sorely.

Albert Fert is a physicist at the University Paris-Sud in Orsay and at UMPh, a joint laboratory of CNRS and Company Thales. e-mail: albert.fert@cnrs-thales.fr 